\title{
Regulatory Function of Histone Modifications in Controlling Rice Gene Expression and Plant Growth
}

\author{
Dao-Xiu Zhou • Yongfeng Hu
}

Received: 6 April 2010 / Accepted: 6 July 2010 / Published online: 29 July 2010

(C) Springer Science+Business Media, LLC 2010

\begin{abstract}
Histone modifications play pivotal roles in chromatin remodeling and gene regulation. Rice genome possesses multiple genes encoding different classes of histone modification enzymes. Specific histone modification patterns in rice are associated with either heterochromatic or euchromatic regions or related to gene expression. Functional studies of several rice genes encoding histone deacetylases and histone methyltransferases and demethylases reveal specific regulators involved in transposon repression, development regulation, and responses to environmental conditions. Functional interplay between rice histone modification regulators in gene regulation and transposon silencing and their implication in rice epigenetic variation are discussed.
\end{abstract}

Keywords Chromatin $\cdot$ Histone acetylation $\cdot$ Methylation . Histone deacetylase $\cdot$ Histone methyltransferase $\cdot$ Histone demethylase - jmjC · SUVH · SIR2 - Epigenetic regulation . Stress inducible

In eukaryotes, the genomic DNA is tightly compacted into a complex structure known as chromatin. To control genome activities, the accessibility of chromatin is dynamically regulated during growth and development. The massive changes in gene expression that occur during developmental transitions rely at least in part on epigenetic processes such as chromatin remodeling to establish specific states for gene expression. In addition, plants are sessile organisms that have to adapt to their living environments. It is therefore essential for plants to develop

D.-X. Zhou $(\bowtie) \cdot Y$. Hu

Institute de Biologie des Plantes, UMR8618, Université Paris-sud 11 ,

91405 Orsay, France

e-mail: dao-xiu.zhou@u-psud.fr rapid responses to changes in environmental conditions for their adaptation and survival. Rapid changes of chromatin structure play a central role in regulating gene expression in response to environmental cues. During the last decade, many plant DNA-binding transcription factors involved in developmental and inducible gene regulations have been identified. However, how these factors activate or repress transcription in a specific chromatin context is not clearly known. Chromatin structure and remodeling are basic components of genetic and epigenetic regulations of genome expression (Horn and Peterson 2002). Nucleosome is the basic structure of chromatin and is composed of 4 types of core histones. Covalent modifications of the Nterminal tails of the core histones play pivotal roles in chromatin remodeling and in gene regulation (Millar and Grunstein 2006). Histone modifications include acetylation, methylation, phosphorylation, ubiquitinylation, and others. Most of the modifications are on the lysine, arginine, or serine residues of $\mathrm{H} 3$ or $\mathrm{H} 4$. Specific patterns of histone modifications determine active or repressed states of the cognate chromatin (Berger 2007). Different chromatin structures define distinct epigenomes which is reflected by specific gene expression pattern of different cell types and responses to different environmental conditions. Histone modification and recognition genes are found to play important roles in many different aspects of developmental, cellular and genetic processes in Arabidopsis. Functional study of rice histone modification and recognition is emerging. Here, we review recent data of rice histone modification regulation and its function in rice gene expression and plant growth and development. Since there is no much information on histone phosphorylation and ubiquitination in rice, the review will focus on the regulatory mechanism of histone acetylation and methylation. 


\section{Histone acetylation in the regulation of rice gene expression}

Among histone modifications, histone lysine acetylation appears to be a dynamic reversible switch for interconversion between permissive and repressive transcriptional states of chromatin domains. Strong acetylation of histones induces relaxation of chromatin structure and is associated with transcriptional activation, whereas weak acetylation leads to chromatin compaction and gene repression (Berger 2007). N-terminal lysine residues of histone $\mathrm{H} 3$ (K9, K14, K18 and K23) and H4 (K5, K8, K12, $\mathrm{K} 16$ and $\mathrm{K} 20$ ) are found to be acetylation/deacetylation targets in Arabidopsis (Earley et al. 2007; Zhang et al. 2007a). The dynamic modulation of histone acetylation in plants is shown to be important for plants to adapt their growth and development to environment changes such as light, temperature, biotic, and abiotic stresses (Chen and Tian 2007; Servet et al. 2010). In rice, acetylation of H3K9 and $\mathrm{H} 4 \mathrm{~K} 12$ is elevated in genes located in euchromatic regions (Yin et al. 2008), suggesting that these markers may be associated with active genes. Dynamic and reversible changes in histone $\mathrm{H} 3$ acetylation and $\mathrm{H} 3 \mathrm{~K} 4$ methylation occur at submergence-inducible genes in rice (Tsuji et al. 2006). When submerged, the rice ADH1 (alcohol dehydrogenase 1) and PDC1 (pyruvate decarboxylase 1) genes are activated in two phases: the first activation occurs after $2 \mathrm{~h}$ of submergence, the second after $12 \mathrm{~h}$. The first induction seems to be associated with tri-methylation of histone $\mathrm{H} 3 \mathrm{~K} 4$ on the $5^{\prime}$ - and $3^{\prime}$-coding regions, while the second induction is correlated with increased histone $\mathrm{H} 3$ acetylation throughout $\mathrm{ADH} 1$ and PDC1 genes. The methylation and acetylation levels return to the initial levels after re-aeration. These data nicely demonstrate the dynamic and reversible changes of histone $\mathrm{H} 3 \mathrm{~K} 4$ methylation and $\mathrm{H} 3$ acetylation in responses to environmental changes in rice. In addition, histone $\mathrm{H} 3 \mathrm{~K} 9$ acetylation is required for the expression of $R I C E$ FLOWERING LOCUS T 1 (RFT1/FT-L3) that is the closest homologue of Heading date $3 a$ (Hd3a) encoding a mobile flowering signal and promote floral transition under short-day conditions (Komiya et al. 2008), showing a role of histone acetylation in the regulation of rice developmental transitions.

\section{Function of histone deacetylases genes in rice}

The homeostatic balance of nucleosomal histone acetylation is maintained by actions of histone acetyltransferases and histone deacetylases (HDAC). Plant HDAC can be grouped into three classes. Among them, two have primary homology to yeast HDAC groups: reduce potassium dependency 3 (RPD3), and silent mating-type information regulation-2 (SIR2; Fig. 1; Pandey et al. 2002). The other group known as the HD2 class is found in plants only (Lusser et al. 1997). During the last years, HDAC function has been most studied in Arabidopsis. One of the Arabidopsis RPD3 type genes, AtHD1 (HDA19) seems to be a polyvalent HDAC involved in many developmental and stimulus-responsive pathways. Down-regulation of the gene induces pleiotropic developmental abnormalities (Tian and Chen 2001). Other Arabidopsis RPD3-like genes are involved in the control of flowering time, root hair production, RNAi-dependent DNA methylation, and nucleolar dominance in allopolyploid hybrids (Wu et al. 2000; Xu et al. 2005; Aufsatz et al. 2002; Pontes et al. 2007). Rice genome contains at least 19 HDAC genes ( $\mathrm{Hu}$ et al. 2009; Fig. 1). Expression and functional studies suggest that individual rice HDAC genes have specific development functions that may be divergent from the Arabidopsis homologues. Expression of rice HDAC genes shows tissue/ organ specificity. For instance, HDA710 was more expressed in germinating and young seedlings as well as in stamens, whereas $H D A 703$ was highly expressed in callus and in imbibed seeds. HDA714 and HDA706 were found to be mainly expressed in shoots and leaves, whereas HDA716 showed a strong expression in developing endosperm and germinating seeds. Most of the HDAC genes are responsive to drought or salt stresses. For instance, two genes ( $H D A 703$ and $H D A 710)$ are induced, while nine others (i.e., HDA701, HDA702, HDA704, HDA705, HDA706, HDA712, HDA714, HDA716, HDT701, and HDT702) are clearly repressed by drought and salt (Hu et al. 2009). Down-regulation of most of HDAC genes may be important for stress-inducible gene expression in rice. These HDACs may repress stressresponsive gene expression in the absence of stress signals. Therefore, histone acetylation/deacetylation switch is suggested to be an important mechanism of short-term gene regulation in plants (Zhou 2009), may be largely involved in stress responses in rice. While most of the HDAC proteins are localized in nucleus, a few of them are localized in the chloroplast (e.g., HDA714) and/or mitochondrion (e.g., HDA714, SRT702; Chung et al. 2009b), suggesting that these HDACs may have specific functions in rice cells, which remain to be determined.

It has been shown that over-expression of $O s H D A C 1$ (HDA702) leads to increased growth rate and altered architecture in transgenic rice (Jang et al. 2003). It is recently shown that OsHDAC1 epigenetically represses the OsNAC6 gene that controls rice seedling root growth (Chung et al. 2009a). OsHDAC1 interacts with the OsNAC6 gene promoter and deacetylates histone $\mathrm{H} 3 \mathrm{~K} 9, \mathrm{~K} 14$ and $\mathrm{K} 18$ and H4K5, K12 and K16. However, over-expression of several other rice HDAC genes does not produce any visible 
Fig. 1 Schematic representation of reversible modifications of histone H3K4, K9, and $\mathrm{K} 27$. Histone $\mathrm{H} 3 \mathrm{~K} 9$ acetylation and $\mathrm{H} 3 \mathrm{~K} 4$ methylation are markers for gene activation. Histone H3K9 acetylation/deacetylation is catalyzed by histone acetyltransferases (HAT) and deacetylases (HDAC) that has three groups (RPD3, HD2 and SIR2). H3K9 methylation is mediated by $\mathrm{Su}$ (var) 3-9 homologues (SUVH), while that of histone $\mathrm{H} 3 \mathrm{~K} 4$ and H3K27 is suggested to be mediated by Tritorax homologues (TXH) and E(Z) homologues, respectively. Histone lysine demethylases LSD1 and jmjC proteins are suggested to be involved in the reversion of methylated $\mathrm{H} 3 \mathrm{~K} 4$, $\mathrm{K} 9$, and K27. Numerals in parentheses are numbers of genes found in rice genome.
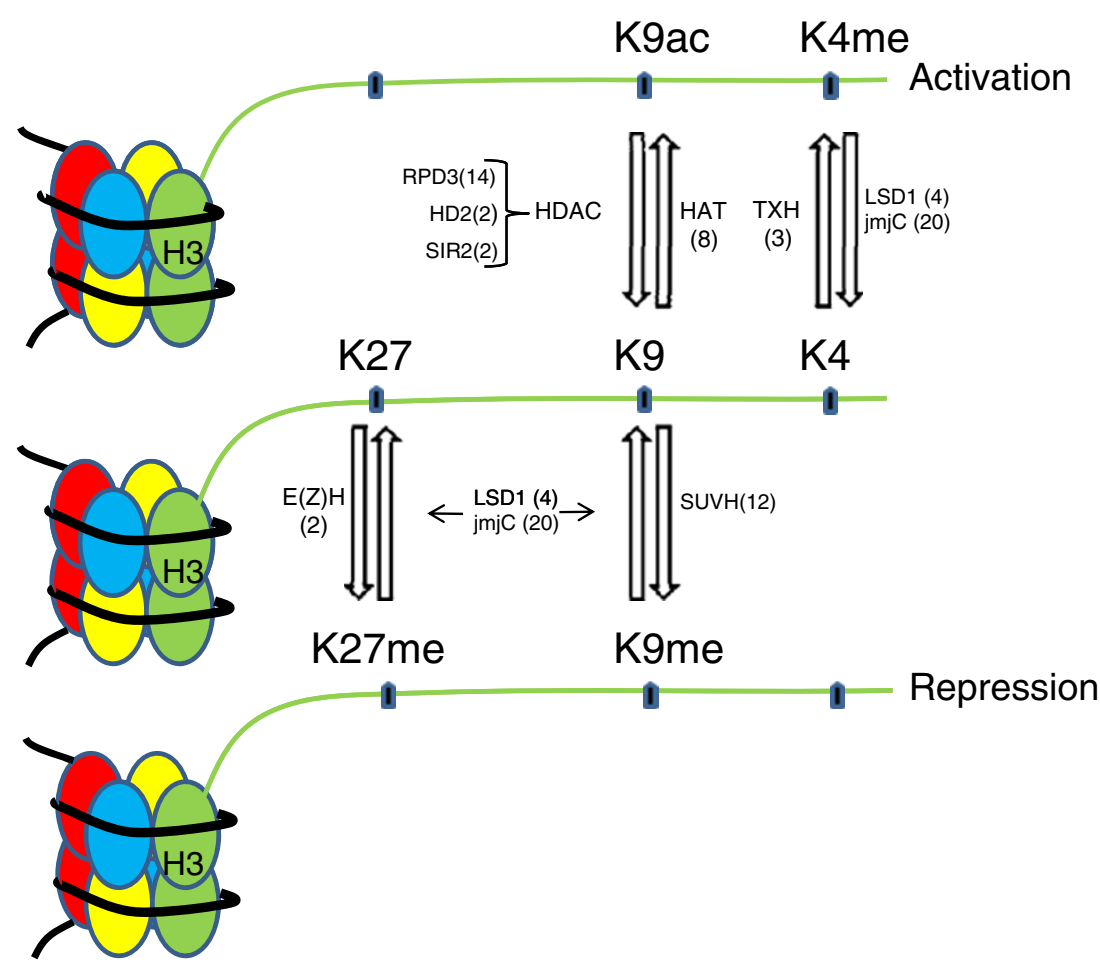

phenotype. In contrast, down-regulation of a few HDAC genes affects different developmental aspects ( $\mathrm{Hu}$ et al. 2009). Although the mechanism by which $H D A C$ are involved in rice development regulation is unclear, those rice genes seem to have divergent developmental functions compared to closely related homologues in Arabidopsis.

SIR2 proteins are $\mathrm{NAD}^{+}$-dependent HDACs, some of which have been found to be involved in increasing lifespan in yeast and animals (reviewed in Finkel et al. 2009). Plant genomes seem to contain relatively fewer SIR 2 homologues than other eukaryotes. In rice or Arabidopsis, only two SIR2 family genes have been identified (Pandey et al. 2002). Phylogenetic analysis of identified plant SIR2 homologues shows that they belong only to two of the four classes of the family, which have only plant and animal members (Huang et al. 2007). As there are fewer SIR2related genes found in plant genomes, important questions arise such as whether plant SIR2-related proteins conserve the similar functions as yeast and animal SIR2 proteins. The expression patterns of the two rice genes (SRT701 and SRT702) are different (Hu et al. 2009). The two proteins are likely to have distinct functions. In addition, SRT701 (also called OsSRT1) is a nuclear protein, while SRT702 seems to be localized in the mitochondria (Huang et al. 2007; Chung et al. 2009b). SRT701 is widely expressed but with higher levels in rapidly dividing tissues. SRT701 RNAi leads to an increase of histone H3K9 acetylation and a decrease of H3K9 dimethylation. SRT701 RNAi induces $\mathrm{H}_{2} \mathrm{O}_{2}$ production, DNA fragmentation, cell death, and lesions mimicking plant hypersensitive responses induced by pathogen attacks, whereas over-expression of the gene enhanced tolerance to oxidative stress (Huang et al. 2007). Many transposons and retrotransposons in addition to genes related to hypersensitive response and/or programmed cell death are activated in the RNAi plants. Histone H3K9 acetylation on transposable elements and some of the hypersensitive response-related genes is increased in the RNAi plants, indicating that transposons and cell deathrelated genes might be amongst the primary targets of SRT701 for histone deacetylation. Therefore, SRT701 may be required for genome stability to ensure plant cell growth, but likely implicating different molecular mechanisms from yeast and animal homologues. These results suggest that histone acetylation is an important component for transposon repression in rice (Fig. 2; see below).

\section{Histone methylation and histone lysine methyltransferases in rice}

Histone lysine methylation is an important epigenetic modification with both activating and repressive roles in gene expression. Histone lysine residues can be mono-, di-, or trimethylated, where each distinct methyl state may have different biological functions (Bannister and Kouzarides 2004). For instance, di- and trimethylation of H3K9 (H3K9me2, H3K9me3) and trimethylation of H3K27 (H3K27me3) negatively correlate with gene expression, 


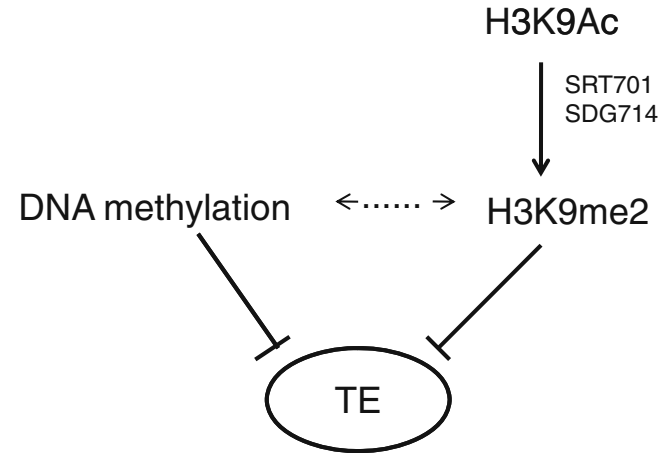

Fig. 2 A model of epigenetic repression of transposable elements (TE) in rice. Both DNA methylation and histone modifications are important components of transposon silencing. Three rice genes are shown to be involved histone H3K9 deacetylation (SRT701) and methylation (SDG714) in transposon repression.

whereas trimethylation of $\mathrm{H} 3 \mathrm{~K} 4(\mathrm{H} 3 \mathrm{~K} 4 \mathrm{me} 3)$, as mentioned above, positively correlate with the expression of target genes (Pfluger and Wagner 2007; Fig. 1). These differently methylated histone lysine residues are recognized by different chromatin protein modules that induce distinct chromatin structures. For instance, H3K9me2 is shown to be associated with Heterchromatin Protein 1 in animal cells (reviewed in Vermaak and Malik 2009), while H3K27me3 is associated with Polycomb group ( $\mathrm{PcG}$ ) repressive complexes in both plant and animal cells (reviewed in Hennig and Derkacheva 2009).

Results from immunostaining and chromatin immunoprecipitation (ChIP) coupled with DNA microarray (chip) (ChIPchip) analysis in Arabidopsis suggest that methylation of $\mathrm{H} 3 \mathrm{~K} 9$ and $\mathrm{H} 3 \mathrm{~K} 27$ is important for chromatin structure and gene regulation. $\mathrm{H} 3 \mathrm{~K} 9 \mathrm{me} 2$ is found to be enriched in heterochromatic repetitive sequence regions, while $\mathrm{H} 3 \mathrm{~K} 9 \mathrm{me} 3$ and $\mathrm{H} 3 \mathrm{~K} 27 \mathrm{me} 3$ are distributed in the $5^{\prime}$ ends of genes in euchromatic regions (Lippman et al. 2004; Turck et al. 2007; Zhang et al. 2007b). The distribution of H3K9me3 and H3K27me3 does not overlap, suggesting that they may respond to different cellular signals or be involved in distinct regulatory pathways. Analysis of H3K4 methylation of two entire chromosomes in rice revealed that half of proteincoding genes have di- and/or trimethylated $\mathrm{H} 3 \mathrm{~K} 4$ ( $\mathrm{Li}$ et al. 2008). Rice genes with predominantly H3K4me3 are actively transcribed, whereas genes with predominantly H3K4me2 were transcribed at moderate levels ( $\mathrm{Li}$ et al. 2008).

Enzymes involved in histone methylation usually contain a motif called SET domain, which is named after three Drosophila genes: $\mathrm{Su}(\mathrm{var}) 3-9$, Enhancer of zeste $(\mathrm{E}(\mathrm{Z}))$ and Trithorax, the mutation of which either enhance or suppress epigenetic mutations. A large number of SET-domain genes are identified in rice and Arabidopsis genomes (Fig. 3). Su (var) 3-9 homologues (SUVH) are found to be mostly involved in H3K9 methylation (Fig. 1). Arabidopsis SUVH protein KYPTONITE (KYP) was discovered in a screen for suppressor of an epigenetic mutation due to hypermethylation of cytosine on the locus of SUPERMAN. Both Arabidopsis and rice SUVH proteins are found to have H3K9 mono- and di-methylation activities. This raises a question whether $\mathrm{H} 3 \mathrm{~K} 9$ me3 exists in plants. $\mathrm{E}(\mathrm{Z})$ homologues which are components of $\mathrm{PcG}$ complexes are responsible for H3K27me3 (Fig. 1). Several homologues of $\mathrm{E}(\mathrm{Z})$ in Arabidopsis (i.e., CURLY LEAF (CLF), SWINGER (SWN), MEDEA (MEA)) which are components of PcG-related complexes (PRC2), are shown to behave as essential regulators of plant developmental transitions (Pien and Grossniklaus 2007). The homologues of $\mathrm{E}(\mathrm{Z})$ and other PRC2 components are also identified in rice and some of them are characterized to have functions in rice development, which seem not be the same as found in Arabidopsis (Luo et al. 2009; Fig. 3). This suggests that rice $\mathrm{PcG}$ proteins may have functional specificities. Whether the rice $\mathrm{E}(\mathrm{Z})$ homologues have the H3K27 methyltransferase activity remains to be determined. Trithorax proteins are a group of methyltransferases for H3K4 methylation (Fig. 1). Arabidopsis TRITHORAXRELATED1, 2 (ATX1, 2) and ATX-Related7 (ATXR7) are shown to be involved in the H3K4 methylation required for the expression of FLOWERING LOCUS C (FLC), a flowering repressor (Pien et al. 2008; Saleh et al. 2008; Tamada et al. 2009). In contrast, two other ATX-Related genes, ATXR5 and ATXR6, are H3K27 monomethyltransferases required for chromatin structure and gene silencing (Jacob et al. 2009). In the rice genome, three thithorax homologues (TXH) are found and their function has not been studied yet (Fig. 3).

Drosophila $\mathrm{Su}(\mathrm{var}) 3-9$ protein was the first identified histone lysine methyltransferase specific for H3K9 (Rea et al. 2000). Multiple Su(var)3-9 methyltransferases have been identified in mammals and are shown to play an important role in chromatin function and development (Sims et al. 2003). In line with the genomic characteristics, plant genome encodes many SUVH genes (Baumbusch et al. 2001; Fig. 3). This may be because a large fraction of the genome is repetitive sequences and extensive heterochromatic silencing processes require different SUVH functions. For instance, Arabidopsis genome contains ten $S U V H$ genes, of which KYP (also known as SUVH4), SUVH5 and SUVH6 are shown to undergo H3K9 methylation in a locus-specific manner (Ebbs et al. 2005; Ebbs and Bender 2006; Jackson et al. 2004). The three Arabidopsis SUVH proteins display mono- or dimethyltransferase activity of histone H3K9 (Ebbs and Bender 2006; Jackson et al. 2004). Rice genome encodes 12 SUVH genes (Fig. 3). One of rice SUVH genes, namely $S D G 714$, is found to be involved in $\mathrm{H} 3 \mathrm{~K} 9 \mathrm{me} 2$ and DNA methylation of Tos 17, a copia-like retrotransposon (Ding et al. 2007). A systematic study of rice SUVH genes revealed that different members display distinct function in histone H3K9 methylation, DNA 
Fig. 3 Phylogenetic relationship of SET domain-containing proteins in Oryza sativa (SDG numbers with three numerals starting with 7) and A. thaliana (SDG numbers with 1 or 2 numerals). All the sequences of SET domain-containing proteins were obtained from ChromDB database (www.chromdb.org). The alignment of these sequences was conducted by the ClustalX program. The SET domain sequences were selected to construct the phylogenetic tree by using MEGA3.1. Only the tree topology is shown. The classification of SET domain proteins was in accordance with that described in Baumbusch et al. (2001). SUVH SU(VAR)3-9 homolog, SUVR SUVH-related, $A S H H$ ASH1 homolog, $E(Z) H \mathrm{E}$ (Z) homolog, TXH tritorax homolog. The TXR (TXH-related) and ASHR (ASH1-Related) groups were not noted because of their truncated SET domains. For the proteins that had been published, there designations in the papers were annotated in the parenthesis behind the

ChromDB name $\left(\mathrm{SDG}^{+}\right.$or $\mathrm{SDG}^{+}$).

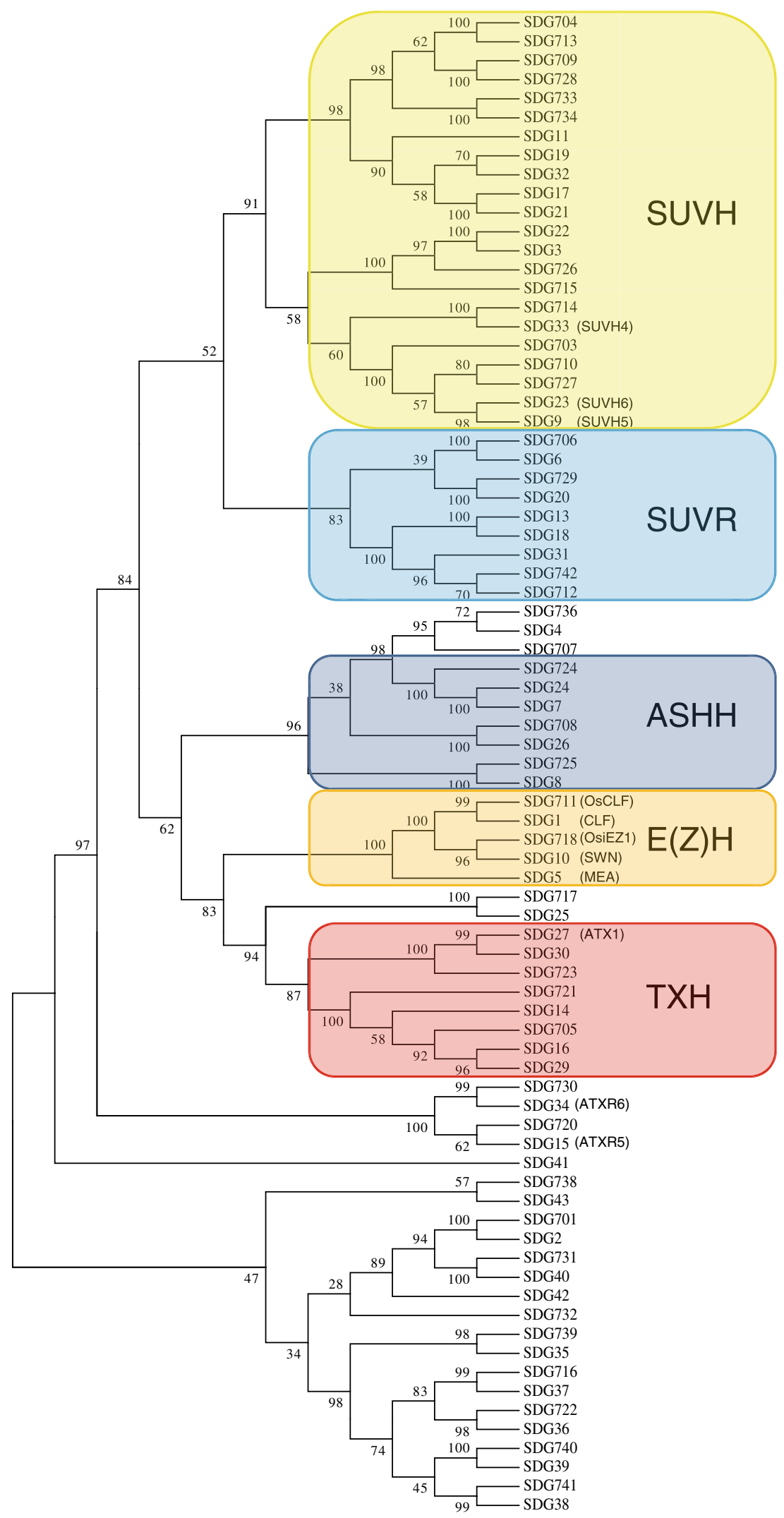


methylation, and transposon silencing (Qin et al. 2010). For instance, down-regulation of most of the rice SUVH members does not induce obvious morphological phenotype, while $S D G 728$ RNAi plants produce deformed seed shape. In addition, both $\mathrm{H} 3 \mathrm{~K} 9 \mathrm{me} 2$ and $\mathrm{H} 3 \mathrm{~K} 9 \mathrm{me} 3$ are decreased in the $S D G 728$ RNAi plants, while down-regulation of other rice SUVH genes (e.g., SDG713) only affects H3K9me2. The expression of Tos17 and a Tyl-copia element is activated in RNAi plants of a few SUVH genes (e.g., SDG703, SDG713 and SDG728), but not affected by downregulation of other SUVH. The activation of Tos 17 is correlated with a clear decrease of H3K9me3 on Tos 17 in SDG728 RNAi plants, suggesting that H3K9me3 may be an important component of retrotransposon silencing.

As described above, down-regulation of the rice HDAC gene SRT701 leads to transcriptional activation of many transposons (Huang et al. 2007). Down-regulation of SRT701 not only augments H3K9ac but also decreases $\mathrm{H} 3 \mathrm{~K} 9 \mathrm{me} 2$. As acetylated $\mathrm{H} 3 \mathrm{~K} 9$ needs to be deacetylated by HDAC before methylation, these data suggest that $S R T 701$ and SUVH genes function within a similar pathway to regulate transposon silencing in rice. Therefore, in addition to DNA methylation that is known to be an important component of transposon and retrotransposnon silencing in plants, histone modification seems to also play a primary role in retrotransposon repression in rice (Fig. 2). Furthermore, SUVH genes (i.e., $S D G 714, S D G 727$, and $S D G 710$ ) are found to have an antagonistic function to the histone H3K9 demethylase gene JMJ706 in regulating H3K9 methylation and panicle development (Qin et al. 2010).

In addition to the SET domain, plant SUVH proteins contain a plant-specific YDG (named after the three conserved amino acids)/SRA (SET- and Ring Finger-associated) domain. The YDG/SRA domain of KYP/SUVH4 is shown to bind directly to methylated DNA (Johnson et al. 2007), suggesting that DNA methylation and H3K9me2 are correlated in Arabidopsis (Soppe et al. 2002; Tariq et al. 2003). Inactivation of rice SDG714 affected DNA methylation on the Tos 17 locus, suggesting that rice SUVH function may be also linked to DNA methylation involved in retrotransposon silencing.

\section{Histone demethylases}

Histone methylation was considered irreversible until recent discovery of histone demethylases (Shi et al. 2004; Cloos et al. 2008). LSD1 was the first histone demethylase to be identified in mammalian cells, and it has been shown to demethylate mono-methylated and di-methylated H3K4 (H3K4mel and $\mathrm{H} 3 \mathrm{~K} 4 \mathrm{me} 2$ ) and H3K9 (H3K9me1 and H3K9me2). In metazoa LSD1 is encoded by a single-copy gene, whereas in rice or Arabidopsis four homologues are found (Fig. 4). Three Arabidopsis LSD1 genes are shown to be involved in

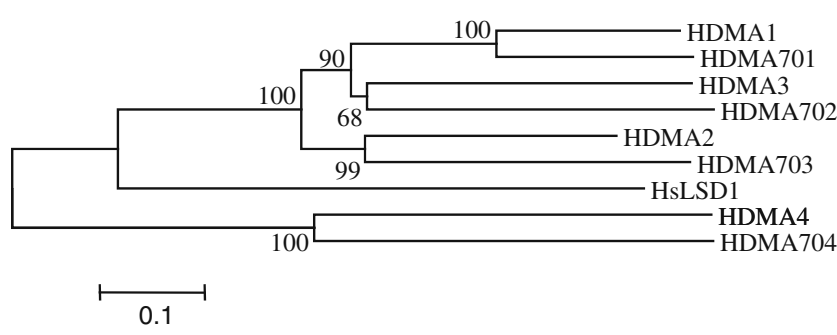

Fig. 4 Phylogenetic relationship of $L S D 1$ genes from rice (HDM701704), Arabidopsis (HDM1-4) and human LSD1.

flowering regulation, the mutation of which induces $\mathrm{H} 3 \mathrm{~K} 4$ hyperacetylation and a decrease in $\mathrm{H} 3 \mathrm{~K} 27 \mathrm{me} 2$ and $\mathrm{H} 3 \mathrm{~K} 9 \mathrm{me} 2$ on the $5^{\prime}$ region of $F L C$ and produce a later flowering phenotype (Jiang et al. 2007). Jumonji C (jmjC) domaincontaining proteins have been found to function also as histone demethylases (Trewick et al. 2005). Different classes of jmjC proteins have specific histone lysine targets (Klose et al. 2006). Analysis of plant jmjC domain genes leads to the identification of seven groups of $\mathrm{jmjC}$ domain-containing proteins on the basis of the jmjC domain and the overall protein domain architecture (Sun and Zhou 2008). Some of the plant jmjC proteins are conserved with mammalian genes. Others seem not to have close animal homologues. Even in the conserved members, plant proteins have specific modules (Sun and Zhou 2008). Plant jmjC proteins involved specifically in the reversion of di- or trimethylated H3K9 or H3K4 have been identified. Mutations in two Arabidopsis jmjC genes, AtJmj4 (also called JMJ14, At4g20400) and EARLY FLOWERING6 (ELF6, JMJ11 or Atjmj1, At5g04240) which are H3K4 demethylases directly repressing the FLOWERING LOCUS T (FT) and an FT homologue TWIN SISTER OF FT (TSF) and preventing precocious flowering in Arabidopsis (Jeong et al. 2009; Yang et al. 2010). Purified recombinant AtJmj4 protein possesses specific demethylase activity for mono-, di- and trimethylated H3K4. Tagged AtJmj4 and ELF6 proteins associate directly with the $F T$ transcription initiation region, a region where the $\mathrm{H} 3 \mathrm{~K} 4 \mathrm{me} 3$ levels are increased most significantly in the mutants (Jeong et al. 2009). Both jmjC proteins belong to different subclasses of jmjC proteins. It is recently shown that JMJ14 affects mobile RNA silencing in an Arabidopsis transgene system. It also enhances asymmetric cytosine $(\mathrm{CHH}, \mathrm{H}=\mathrm{A}, \mathrm{T}$ or $\mathrm{C})$ methylation, abundance of transopson transcripts. These results illustrate a link between RNA silencing and histone H3K4me3 demethylation (Searle et al. 2010). Two different subclasses of mammalian jmjC proteins are also found to be $\mathrm{H} 3 \mathrm{~K} 4$ demethylases. In rice, a jmjC protein (JMJ704) is also identified to have a H3K4 demethylase activity (Peng, J. and Jeong H. J. Functional study of rice jumonji $\mathrm{C}$ domain containing gene JMJ704. Abstract P1-53 presented at The Sixth International Rice genetics Symposium, November 16-19, 2009. Manila www. ricegenetics.com). 
Arabidopsis INCREASED EXPRESSION OF BONSAI METHYLATION1 (IBM1; also known as AtJmj15 or JMJ25, At3g07610), represses genic cytosine methylation, possibly through demethylation of H3K9 (Saze et al. 2008). JMJ706, a rice member of the JMJD2 group of jmjC genes, is shown to encode a heterochromatin-associated protein (Sun and Zhou, 2008). In vitro histone demthylation assays and analysis of TDNA insertion mutants revealed that JMJ706 is involved in H3K9 demethylation required for the expression of a subset of regulatory genes for rice panicle development. However, the mutation of JMJ706 seems not to affect DNA methylation in tandem repeats (Sun 2009). Minor changes in DNA methylation on the retrotransposon Tos 17 is observed. Therefore, different plant jmjC proteins involved in $\mathrm{H} 3 \mathrm{~K}$ 9 demethylation may have distinct function in chromatin regulation.

As mentioned above, inactivation of three SUVH genes by amiRNA in the jmj706 mutant background restore partially the panicle phenotype of jmj706 and histone H3K9 methylation. This group of SUVH methyltransferase genes and JMJ706 appear to be involved in different regulatory pathways, but simultaneous inactivation could compensate for each other. This indicates that interaction between the two antagonistic families of enzymes is collectively involved to maintain histone $\mathrm{H} 3 \mathrm{~K} 9$ methylation homeostasis that is important for rice development.

H3K27me3 are found to be distributed in the $5^{\prime}$ ends of genes in euchromatic regions in Arabidopsis (Turck et al. 2007; Zhang et al. 2007b). Whether H3K27me3 has a similar distribution on rice genome remains to be determined. The repression of many important plant developmental key genes is mediated by $\mathrm{H} 3 \mathrm{~K} 27$ methylation involving $\mathrm{PcG}$ protein complexes. For instance, SET domain-containing PcG proteins CLF and SWN are required for H3K27me3-mediated silencing of important developmental regulatory genes such as the floral organ homeotic gene AGAMOUS during vegetative growth, the meristem regulator SHOOTMERISTEMLESS (STM) in seedlings and the seed development gene PHERES in vegetative tissues (reviewed in Pien and Grossniklaus 2007). The establishment and the maintenance of the repression of the flowering repressor $F L C$ is also mediated by H3K27 methylation. This suggests that H3K27memediated gene silencing may be mainly involved in developmental decision in plants. However, activities involved in the demethylation of $\mathrm{H} 3 \mathrm{~K} 27 \mathrm{me} 3$ remain to be identified in plants.

\section{Conclusions and perspectives}

Current knowledge on the function of histone modification regulation and recognition suggests that there are both conservation and difference between plants and other eukaryotic systems in chromatin mechanism of gene regulation. The sessile lifestyle of plants requires increased developmental plasticity. Histone modification and recognition are essential for programming and reprogramming of plant developmental processes and for rapid responses to environmental cues. Plant genomes contain a large portion of repetitive sequences, the repression of which involves both DNA methylation and histone modifications. In addition, plants have large families of genes involved in histone modifications. Studying specific functions of the individual family members will be needed to understand the complexity of regulation of histone modification and epigenomic dynamics during plant developmental transitions and in responses to environmental conditions. Identifying functional interaction between histone modification regulators and other chromatin or signaling factors (such as chromatin remodeling factors, DNA-binding transcription factors) will be required to provide a mechanistic view on how histone modification integrates cell signals to regulate chromatin structures. Identifying the mechanism of recognition and reading of different histone modification markers constitutes another challenge of chromatin signaling for gene regulation. Rice as a cereal has specific development and growth specificities. Studying the mechanism of establishment, maintenance, and erasure of specific rice epigenomes will be essential to unravel the epigenetic mechanism of rice gene regulation. Progresses in this field in rice will have a great impact on the understanding of genetic and epigenetic basis of cereal growth and adaptation to environmental conditions such as water, light, temperature, soil, and pathogens. It is suggested that interaction between plant genomes of different origins during crosses may generate specific epigenetic mechanisms of gene regulation such as allelic inactivation and genomic imprinting (Ishikawa and Kinoshita 2009). Epigenomes related to rice hybrid vigor have been recently reported (He et al. 2010). Importantly, it is recently shown that parent plants with little genomic DNA sequence variations, but contrasting epigenetic modification (i.e., DNA methylation) can generate a panel of epigenetic recombinant inbred lines showing variation and high heritability over many generations for several important traits in Arabidopsis (Johannes et al. 2009; Reinders et al. 2009). It remains to know whether variations in chromatin modification (both histone modification and DNA methylation) will be useful as a resource to create novel epigenetic variations controlling important agronomical traits that could be exploited for rice breeding.

\section{References}

Aufsatz W, Mette MF, van der Winden J, Matzke M, Matzke AJ. HDA6, a putative histone deacetylase needed to enhance DNA methylation induced by double-stranded RNA. Embo J. 2002;21:6832-41.

Bannister AJ, Kouzarides T. Histone methylation: recognizing the methyl mark. Methods Enzymol. 2004;376:269-88. 
Baumbusch LO, Thorstensen T, Krauss V, Fischer A, Naumann K, Assalkhou R, et al. The Arabidopsis thaliana genome contains at least 29 active genes encoding SET domain proteins that can be assigned to four evolutionarily conserved classes. Nucleic Acids Res. 2001;29:4319-33.

Berger SL. The complex language of chromatin regulation during transcription. Nature. 2007;447:407-12.

Chen ZJ, Tian L. Roles of dynamic and reversible histone acetylation in plant development and polyploidy. Biochim Biophys Acta. 2007;1769:295-307.

Chung PJ, Kim YS, Jeong JS, Park SH, Nahm BH, Kim JK. The histone deacetylase OsHDAC1 epigenetically regulates the OsNAC6 gene that controls seedling root growth in rice. Plant J. 2009a;59:764-76.

Chung PJ, Kim YS, Park SH, Nahm BH, Kim JK. Subcellular localization of rice histone deacetylases in organelles. FEBS Lett. 2009b;583:2249-54

Cloos PA, Christensen J, Agger K, Helin K. Erasing the methyl mark: histone demethylases at the center of cellular differentiation and disease. Genes Dev. 2008;22:1115-40.

Ding Y, Wang X, Su L, Zhai J, Cao S, Zhang D, et al. SDG714, a histone H3K9 methyltransferase, is involved in Tos17 DNA methylation and transposition in rice. Plant Cell. 2007;19:9-22.

Earley KW, Shook MS, Brower-Toland B, Hicks L, Pikaard CS. In vitro specificities of Arabidopsis co-activator histone acetyltransferases: implications for histone hyperacetylation in gene activation. Plant J. 2007;52:615-26.

Ebbs ML, Bender J. Locus-specific control of DNA methylation by the Arabidopsis SUVH5 histone methyltransferase. Plant Cell. 2006;18:1166-76.

Ebbs ML, Bartee L, Bender J. H3 lysine 9 methylation is maintained on a transcribed inverted repeat by combined action of SUVH6 and SUVH4 methyltransferases. Mol Cell Biol. 2005;25:10507-15.

Finkel T, Deng CX, Mostoslavsky R. Recent progress in the biology and physiology of sirtuins. Nature. 2009;460:587-91.

He G, Zhu X, Elling AA, Chen L, Wang X, Guo L, et al. Global epigenetic and transcriptional trends among two rice subspecies and their reciprocal hybrids. Plant Cell. 2010;22:17-33.

Hennig L, Derkacheva M. Diversity of polycomb group complexes in plants: same rules, different players? Trends Genet. 2009;25:414-23.

Horn PJ, Peterson CL. Molecular biology. Chromatin higher order folding — wrapping up transcription. Science. 2002;297:1824-7.

Hu Y, Qin F, Huang L, Sun Q, Li C, Zhao Y, et al. Rice histone deacetylase genes display specific expression patterns and developmental functions. Biochem Biophys Res Commun. 2009;388:266-71.

Huang L, Sun Q, Qin F, Li C, Zhao Y, Zhou DX. Down-regulation of a SILENT INFORMATION REGULATOR2-related histone deacetylase gene, OsSRT1, induces DNA fragmentation and cell death in rice. Plant Physiol. 2007;144:1508-19.

Ishikawa R, Kinoshita T. Epigenetic programming: the challenge to species hybridization. Mol Plant. 2009;2:589-99.

Jackson JP, Johnson L, Jasencakova Z, Zhang X, PerezBurgos L, Singh PB, et al. Dimethylation of histone H3 lysine 9 is a critical mark for DNA methylation and gene silencing in Arabidopsis thaliana. Chromosoma. 2004;112:308-15.

Jacob Y, Feng S, LeBlanc CA, Bernatavichute YV, Stroud H, Cokus $\mathrm{S}$, et al. ATXR5 and ATXR6 are H3K27 monomethyltransferases required for chromatin structure and gene silencing. Nat Struct Mol Biol. 2009;16:763-8.

Jang IC, Pahk YM, Song SI, Kwon HJ, Nahm BH, Kim JK. Structure and expression of the rice class-I type histone deacetylase genes OsHDAC1-3: OsHDAC1 overexpression in transgenic plants leads to increased growth rate and altered architecture. Plant J. 2003;33:531-41.
Jeong JH, Song HR, Ko JH, Jeong YM, Kwon YE, Seol JH, et al. Repression of FLOWERING LOCUS T chromatin by functionally redundant histone H3 lysine 4 demethylases in Arabidopsis. PLoS One. 2009;4:e8033.

Jiang D, Yang W, He Y, Amasino RM. Arabidopsis relatives of the human lysine-specific Demethylase1 repress the expression of FWA and FLOWERING LOCUS C and thus promote the floral transition. Plant Cell. 2007;19:2975-87.

Johannes F, Porcher E, Teixeira FK, Saliba-Colombani V, Simon M, Agier $\mathrm{N}$, et al. Assessing the impact of transgenerational epigenetic variation on complex traits. PLoS Genet. 2009;5: e1000530.

Johnson LM, Bostick M, Zhang X, Kraft E, Henderson I, Callis J, et al. The SRA methyl-cytosine-binding domain links DNA and histone methylation. Curr Biol. 2007;17:379-84.

Klose RJ, Kallin EM, Zhang Y. JmjC-domain-containing proteins and histone demethylation. Nat Rev Genet. 2006;7:715-27.

Komiya R, Ikegami A, Tamaki S, Yokoi S, Shimamoto K. Hd3a and RFT1 are essential for flowering in rice. Development. 2008;135:767-74.

Li X, Wang X, He K, Ma Y, Su N, He H, et al. High-resolution mapping of epigenetic modifications of the rice genome uncovers interplay between DNA methylation, histone methylation, and gene expression. Plant Cell. 2008;20:259-76.

Lippman Z, Gendrel AV, Black M, Vaughn MW, Dedhia N, McCombie WR, et al. Role of transposable elements in heterochromatin and epigenetic control. Nature. 2004;430:471-6.

Luo M, Platten D, Chaudhury A, Peacock WJ, Dennis ES. Expression, imprinting, and evolution of rice homologs of the polycomb group genes. Mol Plant. 2009;2:711-23.

Lusser A, Brosch G, Loidl A, Haas H, Loidl P. Identification of maize histone deacetylase HD2 as an acidic nucleolar phosphoprotein. Science. 1997;277:88-91.

Millar CB, Grunstein M. Genome-wide patterns of histone modifications in yeast. Nat Rev Mol Cell Biol. 2006;7:657-66.

Pandey R, Muller A, Napoli CA, Selinger DA, Pikaard CS, Richards EJ, et al. Analysis of histone acetyltransferase and histone deacetylase families of Arabidopsis thaliana suggests functional diversification of chromatin modification among multicellular eukaryotes. Nucleic Acids Res. 2002;30:5036-55.

Pfluger J, Wagner D. Histone modifications and dynamic regulation of genome accessibility in plants. Curr Opin Plant Biol. 2007;10:645-52.

Pien S, Grossniklaus U. Polycomb group and trithorax group proteins in Arabidopsis. Biochim Biophys Acta. 2007;1769:375-82.

Pien S, Fleury D, Mylne JS, Crevillen P, Inze D, Avramova Z, et al. ARABIDOPSIS TRITHORAX1 dynamically regulates FLOWERING LOCUS C activation via histone 3 lysine 4 trimethylation. Plant Cell. 2008;20:580-8.

Pontes O, Lawrence RJ, Silva M, Preuss S, Costa-Nunes P, Earley K, et al. Postembryonic establishment of megabase-scale gene silencing in nucleolar dominance. PLoS One. 2007;2:e1157.

Qin F, Sun Q, Huang L, Chen X, Zhou D-X. Rice SUVH Histone Methyltransferase Genes Display Specific Functions in Chromatin Modification and Retrotransposon Repression. Mol Plant. 2010 (in press)

Rea S, Eisenhaber F, O'Carroll D, Strahl BD, Sun ZW, Schmid M, et al. Regulation of chromatin structure by site-specific histone $\mathrm{H3}$ methyltransferases. Nature. 2000;406:593-9.

Reinders J, Wulff BB, Mirouze M, Mari-Ordonez A, Dapp M, Rozhon $\mathrm{W}$, et al. Compromised stability of DNA methylation and transposon immobilization in mosaic Arabidopsis epigenomes. Genes Dev. 2009;23:939-50.

Saleh A, Alvarez-Venegas R, Avramova Z. Dynamic and stable histone $\mathrm{H} 3$ methylation patterns at the Arabidopsis FLC and AP1 loci. Gene. 2008;423:43-7. 
Saze H, Shiraishi A, Miura A, Kakutani T. Control of genic DNA methylation by a jmjC domain-containing protein in Arabidopsis thaliana. Science. 2008;319:462-5.

Searle IR, Pontes O, Melnyk CW, Smith LM, Baulcombe DC. JMJ14, a JmjC domain protein, is required for RNA silencing and cell-tocell movement of an RNA silencing signal in Arabidopsis. Genes Dev. 2010;24:986-91.

Servet C, Conde e Silva N, Zhou D-X. Histone acetyltransferase AtGCN5/ HAG1 is a versatile regulator of developmental and inducible gene expression in Arabidopsis. Mol Plant. 2010 (in press)

Shi Y, Lan F, Matson C, Mulligan P, Whetstine JR, Cole PA, et al. Histone demethylation mediated by the nuclear amine oxidase homolog LSD1. Cell. 2004;119:941-53.

Sims 3rd RJ, Nishioka K, Reinberg D. Histone lysine methylation: a signature for chromatin function. Trends Genet. 2003;19:629-39.

Soppe WJ, Jasencakova Z, Houben A, Kakutani T, Meister A, Huang $\mathrm{MS}$, et al. DNA methylation controls histone $\mathrm{H} 3$ lysine 9 methylation and heterochromatin assembly in Arabidopsis. Embo J. 2002;21:6549-59.

Sun Q, Zhou DX. Rice jmjC domain-containing gene JMJ706 encodes H3K9 demethylase required for floral organ development. Proc Natl Acad Sci U S A. 2008;105:13679-84.

Sun QR. Rice histone modification enzyme studies. PhD Thesis, Huazhong Agricultural University, Wuhan, China; 2009.

Tamada Y, Yun JY, Woo SC, Amasino RM. ARABIDOPSIS TRITHORAX-RELATED7 is required for methylation of lysine 4 of histone $\mathrm{H} 3$ and for transcriptional activation of FLOWERING LOCUS C. Plant Cell. 2009;21:3257-69.

Tariq M, Saze H, Probst AV, Lichota J, Habu Y, Paszkowski J. Erasure of $\mathrm{CpG}$ methylation in Arabidopsis alters patterns of histone $\mathrm{H} 3$ methylation in heterochromatin. Proc Natl Acad Sci U S A. 2003;100:8823-7.

Tian L, Chen ZJ. Blocking histone deacetylation in Arabidopsis induces pleiotropic effects on plant gene regulation and development. Proc Natl Acad Sci U S A. 2001;98:200-5.
Trewick SC, McLaughlin PJ, Allshire RC. Methylation: lost in hydroxylation? EMBO Rep. 2005;6:315-20.

Tsuji H, Saika H, Tsutsumi N, Hirai A, Nakazono M. Dynamic and reversible changes in histone $\mathrm{H} 3$-Lys4 methylation and $\mathrm{H} 3$ acetylation occurring at submergence-inducible genes in rice. Plant Cell Physiol. 2006;47:995-1003.

Turck F, Roudier F, Farrona S, Martin-Magniette ML, Guillaume E, Buisine N, et al. Arabidopsis TFL2/LHP1 specifically associates with genes marked by trimethylation of histone H3 lysine 27. PLoS Genet. 2007;3:e86.

Vermaak D, Malik HS. Multiple roles for heterochromatin protein 1 genes in Drosophila. Annu Rev Genet. 2009;43:467-92.

Wu K, Malik K, Tian L, Brown D, Miki B. Functional analysis of a RPD3 histone deacetylase homologue in Arabidopsis thaliana. Plant Mol Biol. 2000;44:167-76.

Xu CR, Liu C, Wang YL, Li LC, Chen WQ, Xu ZH, et al. Histone acetylation affects expression of cellular patterning genes in the Arabidopsis root epidermis. Proc Natl Acad Sci U S A. 2005;102:14469-74.

Yang W, Jiang D, Jiang J, He Y. A plant-specific histone H3 lysine-4 demethylase represses the floral transition in Arabidopsis. Plant J. 2010;62:663-73.

Yin BL, Guo L, Zhang DF, Terzaghi W, Wang XF, Liu TT, et al. Integration of cytological features with molecular and epigenetic properties of rice chromosome 4. Mol Plant. 2008;1:816-29.

Zhang K, Sridhar VV, Zhu J, Kapoor A, Zhu JK. Distinctive core histone post-translational modification patterns in Arabidopsis thaliana. PLoS One. 2007a;2:e1210.

Zhang X, Germann S, Blus BJ, Khorasanizadeh S, Gaudin V, Jacobsen SE. The Arabidopsis LHP1 protein colocalizes with histone H3 Lys27 trimethylation. Nat Struct Mol Biol. 2007b;14:869-71.

Zhou DX. Regulatory mechanism of histone epigenetic modifications in plants. Epigenetics. 2009;4:15-8. 